\title{
Animal Testing
}

National Cancer Institute

\section{Source}

National Cancer Institute. Animal Testing. NCI Thesaurus. Code C16087.

The use of animals for testing the safety and efficacy of drugs or treatments. 